\title{
Coupled experimental/numerical workflow for assessing quantitative diagenesis and dynamic porosity/permeability evolution in calcite-cemented sandstone reservoir rocks
}

\author{
Steven Claes ${ }^{1}$, Fadi H. Nader ${ }^{2}{ }^{*}$, and Souhail Youssef ${ }^{2}$ \\ ${ }^{1}$ Department of Civil Engineering, Building Physics Section, KU Leuven University, Leuven, Belgium \\ ${ }^{2}$ Division of Geosciences, IFP Energies nouvelles, 1-4, avenue de Bois-Préau, 92852 Rueil-Malmaison Cedex, France
}

Received: 17 April 2018 / Accepted: 27 June 2018

\begin{abstract}
Some of the world best hydrocarbon reservoirs (carbonates and siliciclastics) are also believed to be valuable for subsurface storage of $\mathrm{CO}_{2}$ and other fluids. Yet, these reservoirs are heterogeneous in terms of their mineralogy and flow properties, at varying spatial-temporal scales. Therefore, predicting the porosity and permeability (flow properties) evolution of carbonates and sandstones remains a tedious task. Diagenesis refers to the alteration of sedimentary rocks through geologic time, mainly due to rock-fluid interactions. It affects primarily the flow properties (porosity and permeability) of already heterogeneous reservoir rocks. In this project a new approach is proposed to calculate/quantify the influence of diagenetic phases (e.g. dissolution, cement plugging) on flow properties of typical sandstone reservoir rocks (Early Jurassic Luxembourg Formation). A series of laboratory experiments are performed in which diagenetic phases (e.g. pore blocking calcite cement in sandstone) are selectively leached from pre-studied samples, with the quantification of the petrophysical characteristics with and without cement to especially infer permeability evolution. Poorly and heavily calcite-cemented sandstone samples, as well as some intermediate cemented samples were used. The results show a distinctive dissolution pattern for different cementation grades and varying Representative Elementary Volumes (REVs). These conclusions have important consequences for upscaling diagenesis effects on reservoirs, and the interpretation of geochemical modelling results of diagenetic processes. The same approach can be applied on other type of cements and host-rocks, and could be improved by integrating other petrophysical analyses (e.g. petroacoustic, NMR).
\end{abstract}

\section{Introduction}

Heterogeneity is an inherent characteristic of reservoir rocks, which is determined by their geological evolution, e.g. sedimentary origin, diagenetic processes and burial history (Nader, 2016, and references therein). These processes relate to the economic relevance of reservoir rocks. The ultimate goal is to build reliable reservoir models that can be used as guiding tools in the decision process of important strategic and economic questions concerning reservoirs. Hence a better estimation of the production capability of complex reservoir rocks requires a profound understanding of how diagenetic processes affect the pore network (e.g. Algive et al., 2012; Van der Land et al., 2013; Nader, 2016).

The accurate characterization of reservoir rocks requires a quantitative geometric description of the complex microstructure of the rocks and the use of exact or approximate solutions of the equations of motion that govern

*Corresponding author: fadi-henri.nader@ifpen.fr transport phenomena of interest (Claes, 2015). Diagenesis can strongly modify the pore network by different processes such as cementation, dissolution and replacement (e.g. De Boever et al., 2012; Nader, 2016).

For this contribution, carbonate cementation is taken as a case study of diagenetic influence on reservoir rocks. The rock samples used in this study are Early Jurassic sandstones - calcite cemented - of the Luxembourg Formation, from the Lorraine region of southern Belgium, northern France, and the Grand-Duchy of Luxembourg (Fig. 1). The cementation frequently results in laterally continuous or/and non-continuous calcite cemented zones (see also Van Den Bril and Swennen, 2008). A profound knowledge on the origin, distribution and geometry of carbonate cementation is of prime importance in reservoir characterization, since extensively cemented zones may act as a barrier for fluid flow. For example, they can even lead to reservoir compartmentalisation (Gibbons et al., 1993; Prosser et al., 1993). As stated above this will have great influence on the petrophysical characterization of the potential reservoir 
(e.g. Deschamps et al., 2012). However, the correct quantification of these parameters is often omitted in traditional geological studies.

Due to progress in X-ray Computed Tomography (CT), a rapid, non-destructive, high resolution 2D and 3D examination and analysis of almost any kind of material, including earth and soil materials are currently available in practice (e.g. Remeysen and Swennen, 2008). The reconstructed and analysed CT images provide information on the X-ray attenuation of the composing minerals, which is mainly depending on their intrinsic density and atomic number, at resolutions depending on sample size, focal spot size of the X-ray source and the resolution of the X-ray detector (Youssef et al., 2007). Due to recent optimization of X-ray sources and detectors, flexible, multifunctional, high-resolution X-ray CT scanners have been developed allowing 3D quantification on a micron scale, which is required for the study of pore structure and for the determination of matrix characteristics (Youssef et al., 2008, 2013; Mostaghimi et al., 2013).

This study proposes a new approach to obtain experimental data in real time that can be used to better understand the evolution in time of the petrophysical characteristics of the diagenetic process of dissolution (and inversely cementation). This new experimental protocol allows us to accurately quantify the changes on the pore network by dissolution due to reactive fluid percolation of the studied rock. The methodology combines continuous chemical analysis of the fluid (PH measurement) as well as measurement of the permeability and porosity distribution in the sample. Additionally, high resolution CT scans before and after the experiment allow to visualize the changes in the pore network.

\section{Materials and settings}

\subsection{Geological background}

The rock samples used in this study are sandstone of the Luxembourg Formation, which was deposited during the Hettangian and Sinemurian (Early Jurassic) in the Lorraine region of southern Belgium and northern France, and the Grand-Duchy of Luxembourg. This sand-body is deposited as an offshore sand wave complex in a transgressive regime (Van Den Bril and Swennen, 2008; Fig. 1). The depositional area was located in a gulf at the north-eastern border of the Paris Basin (Mertens et al., 1983). This Gulf developed along a NE-SW oriented subsidence direction. Towards the north, the gulf was bordered by the Ardennes and the Rheinisch massif. In the east, the depositional area was bounded by the Variscan Sierck massif as illustrated in Figure 1.

\subsection{Sedimentological characteristics}

The sedimentological characteristics of the Luxembourg Sandstone Formation were extensively studied and unravelled by the work of different authors, which was based principally on palaeocurrent analysis (Monteyne, 1960; Bintz \& Muller, 1965; Mertens et al., 1983; Berners,
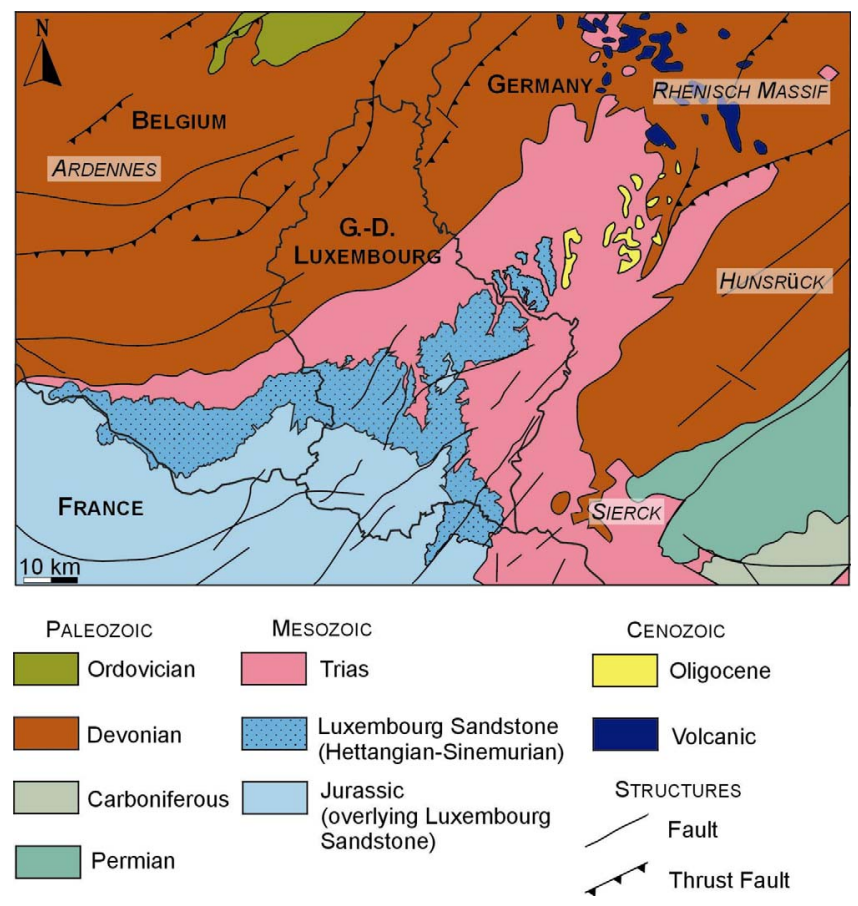

Fig. 1. Simplified geological map of the sample area with location of the main stratigraphic unit and structural lineaments (modified after the geological maps from Belgium, G.D.Luxembourg and France; Van Den Bril and Swennen, 2008).

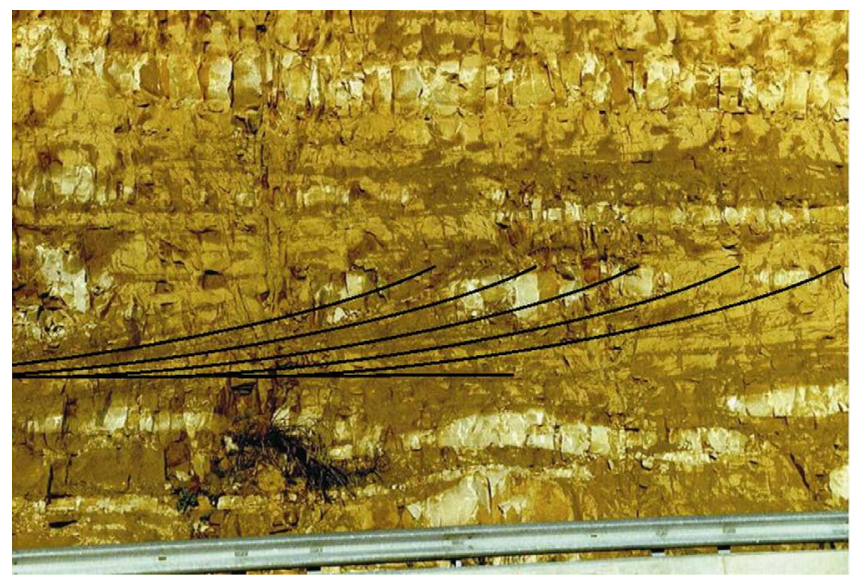

Fig. 2. Outcrop photograph of the calcite cemented lenses (white colour) in the cross-bedded sandstones (Luxembourg Formation) - for more related information check Van Den Bril and Swennen (2008).

1985). The Luxembourg Sandstone Formation can be interpreted as a storm and tide controlled sand wave complex (Mertens et al., 1983), with significant calcite cement. Figure 2 depicts a detailed image of the geometry of the calcite cemented lenses in the cross-bedded sandstone unit. This figure illustrates the sharp lateral contrast between the cemented and uncemented zones. The cements are patchy distributed and probably have nucleated on echinoderm fragments (Van Den Bril and Swennen, 2008). 


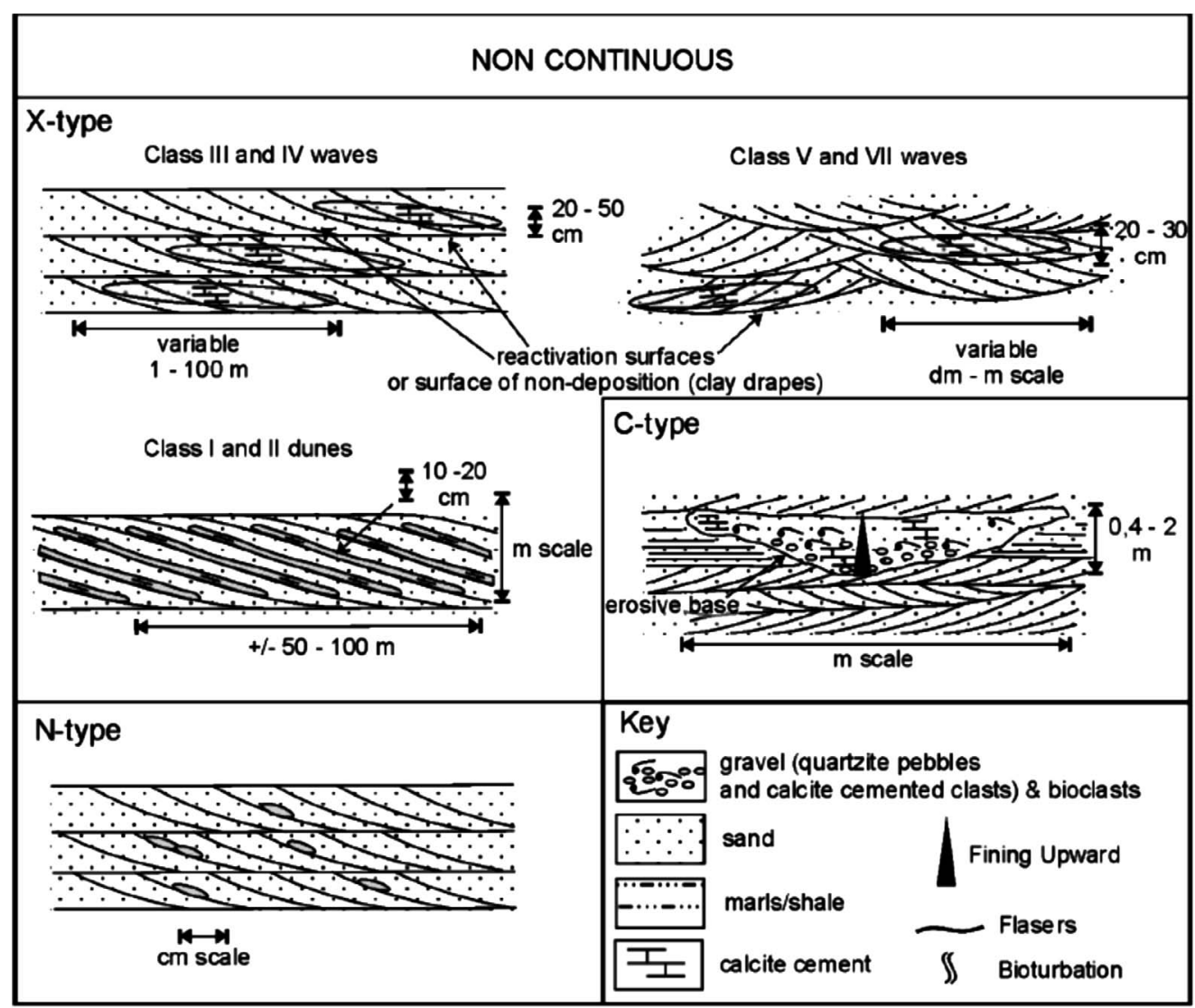

Fig. 3. Various types of calcite cementation geometry in the Luxembourg Sandstone Formation. The sand wave classes correspond to the classification from Allen (1980).

Van Den Bril and Swennen (2008) demonstrated that the calcite cement in the Luxembourg sandstone Formation is predominantly controlled by the original sedimentary architecture (as invoked above). Cement occurring in non-continuous lenses can be subdivided based on their configuration and the type of waves that deposited the matrix material. Cementation in the cross-bedded sands occurs parallel to the bedding (Fig. 2). The cemented lenses generally have a thickness of 20 to $50 \mathrm{~cm}$, and their length varies from 1 to $10 \mathrm{~m}$ depending on the sand wave class of the cross bedded zone. Larger lenses (up to $100 \mathrm{~m}$ ) are less frequently observed. The lenses are bounded at the base by reactivation surfaces or fine grained sediments indicating periods of lower sedimentation rates or non-deposition. Based on the characteristics of the cross-bedded units, further subdivision can be carried out by use of Allen's classification of sand waves (Allen, 1980). Three main subtypes of resulting calcite cement geometry involve (Fig. 3): i) class II and IV subtypes; ii) class V and VI subtypes; and iii) sand dune subtypes (class I and II). These subtypes are defined by the influence of reversing currents on the crossbedded sands. The grain size distribution shows that cemented lenses are located in the coarser and better sorted part of the cross-bed. The uncemented zones contain a higher amount of finer grained particles (Van Den Bril and Swennen, 2008).

\subsection{Petrophysical characteristics}

Small plug samples with a diameter of $1 \mathrm{~cm}$ and a height of $2 \mathrm{~cm}$ are drilled out of hand-specimens. The height of the cores is always taken perpendicular to the bedding. Using the results of the sedimentological analysis, the samples can be subdivided in three different groups: well cemented, intermediate cemented, and uncemented (or poorly cemented) samples. Porosity is measured by analysing the weight difference between the dry and water saturated states of the samples. The vertical permeability is determined by measuring the pressure difference $(\Delta P[\mathrm{~Pa}])$ between the top and bottom of plug for different flow rates according to an internal protocol of IFPEN based on the Darcy's law. By using different flow rates, the accuracy of the measurement is increased and confidence bounds on the measurement can be established.

$$
K(t)=-\frac{\mu L Q}{S \Delta P(t)}
$$

With $L[\mathrm{~m}]$ representing the height of the plug, $S\left[\mathrm{~m}^{2}\right]$ the cross section area and $\mu[\mathrm{Pa} \mathrm{s}]$ the dynamic viscosity of the used fluid.

The uncemented samples have a bulk porosity of around $21 \%$ and the vertical permeability varies between 374 and $476 \mathrm{mD}$. The bulk porosity values of the cemented 
and transition samples vary between 5 and $12.5 \%$, respectively. The permeability of these samples is much lower, typically below $10 \mathrm{mD}$, compared to the uncemented samples and in some cases no permeability could be determined because the sample fractured due to pressure build-up during the measurements.

\section{Methods}

\subsection{Quantified petrography and petrophysics}

A dozen of representative samples of the Luxembourg Sandstone Formation (see above) were provided from previous work that have been achieved by Van Den Bril and Swennen (2008) - courtesy of Prof. Rudy Swennen (KU Leuven University, Belgium). Those samples with a varying degree of calcite cementation were selected for further investigation. They were impregnated with yellow epoxy to identify the pore space, and stained with alizarin red-S and potassium ferricyanide to differentiate carbonate minerals also based on their ferroan nature (Dickson, 1966). Petrographic analyses under conventional polarized microscope, fluorescence (Nikon Eclipse LV100 POL microscope) and cathodoluminescence (CL, NIKON eclipse ME600) consisted firstly of characterizing the overall components, matrix phases and porosity distribution of the investigated thin sections. All thin sections were scanned and analyzed with JMicroVision@ (version 1.2.7, Roduit, 2007) to quantify porosity, amount of grains and different kinds of cement for every thin section by point counting. Matlab ${ }^{\circledR}$ scripts, developed by Claes et al. (2016), were used to determine porosity, overall cement and grain percentages for each thin section, based on segmentation and digital point counting (500 points, 1000 simulations for each thin section). Both scripts were run with Matlab version 7.9.0.529 (R2009b) Copyright ${ }^{\circledR}$ 1984-2013 and make use of the Matlab Image Processing Toolbox ${ }^{\mathrm{TM}}$.

Micro-Computed Tomography $(\mu \mathrm{CT})$ allowed to carry out a 3D quantitative and qualitative investigation of the pore space at a high resolution. In this study, this technique was used to determine the interconnectivity of pores. Three samples were scanned with $\mu \mathrm{CT}$ : i.e. poorly calcite cemented, heavily calcite cemented, and mixed (poor/heavy cemented) transitional sandstones samples. The $\mu \mathrm{CT}$ equipment installed at IFPEN is a microtomograph X Nanotom from PHOENIX X-ray (German made). A Hamamatsu flat detector $(110 \times 110 \mathrm{~mm})$ was used in the setup.

He-injection porosity and classical water-saturation permeability measurements were applied on the same samples that were analyzed by MICP (since the latter is a destructive technique). Mercury Injection Capillary Pressure (MICP) measurements were performed at IFPEN and provide porosity \%-values and permeability. MICP was executed with a AutoPore IV of Micromeritics Instrument Corporation. This mercury porosimeter of 60000 psi ( 4000 bar) covers a pore throat size range of approximately $0.003-360 \mu \mathrm{m}$. It involves the intrusion of mercury (i.e. a non-wetting liquid) at a high pressure, and constant increasing rate, into the investigated sample. The injected volume allows the determination of the pore throat and bulk $(\mathrm{Hg})$ porosity. Permeability values were also calculated based on Swanson's empirical equation correlating permeability with MICP (Swanson, 1981).

\subsection{Experimental dissolution setup}

A new experimental device was specially engineered at IFPEN to simulate fluid flow in reservoir rock samples. The setup consists in a motorized dual pump system that can produce flow through the sample and a confinement pump that is used to generate the desired confinement pressure around the sample to assure no runoff from the sides can influence the measurements (Fig. 4). The pumps are equipped with displacement recorders which allow an accurate control of the flow rate. The inlet cup is equipped with a spiral to ensure a homogeneous injection of the percolating fluid over the bottom surface of the sample. A PH meter is added at the outlet. This configuration allows to gather chemical information about the change of reactive fluids during the experiment.

The whole system is installed in an X-ray chamber and the sample can turn around its axis. This allows to make $\mu$-Ct scans before and after the experiment and radiographs every $2 \mathrm{~s}$ during the experiment. Scans are performed at $70 \mathrm{Kv}$ tube voltage and $100 \mu \mathrm{A}$ current. The scans before and after the dissolution have a resolution of $6 \mu \mathrm{m}$ and visualize the middle part of the plug. The radiographs taken during the experiment have a resolution of $12 \mu \mathrm{m}$. This allows to visualize the plug sample in its totality.

\subsection{Image analysis workflow}

Image analysis forms a crucial step in analysing the diagenetic phases in 2D as well as 3D. The workflow consists of: (i) visualizing, segmenting (dual thresholding) and quantifying the porosity and other diagenetic phases that are distinguishable in the used images; (ii) calculating the Representative Elementary Volume (REV); and (iii) labelling and visualizing the segmented images or volumes in $2 \mathrm{D}$ and $3 \mathrm{D}$, respectively. Image treatment is carried out using the Avizo Fire software package (Version 9.1, VSG) and in-house developed scripts ( $c f$. De Boever et al., 2012).

In order to differentiate gas pore from solid matrix voxels, image segmentation needs to be performed. Quantitative analysis of the porosity requires a voxel by voxel determination of pore or solid phases. For segmentation an in-house dual-thresholding algorithm is used. This method is an adaptation of the single-threshold approach, which typically selects pixels/voxels on the basis of their unique histogram range. However, manually determining the boundary values is not straightforward in the case of insufficiently ideal histograms, with peaks that are not obviously separated. The applied dual or hysteresis thresholding uses two intervals of the histogram in order to determine the segmentation (Fig. 5). Voxels corresponding to the first "strong" threshold are classified as foreground voxels, while voxels selected by the second threshold are only considered foreground if they are connected to voxels already selected by the "strong" threshold. The advantages of this algorithm are the reduced sensitivity to residual 


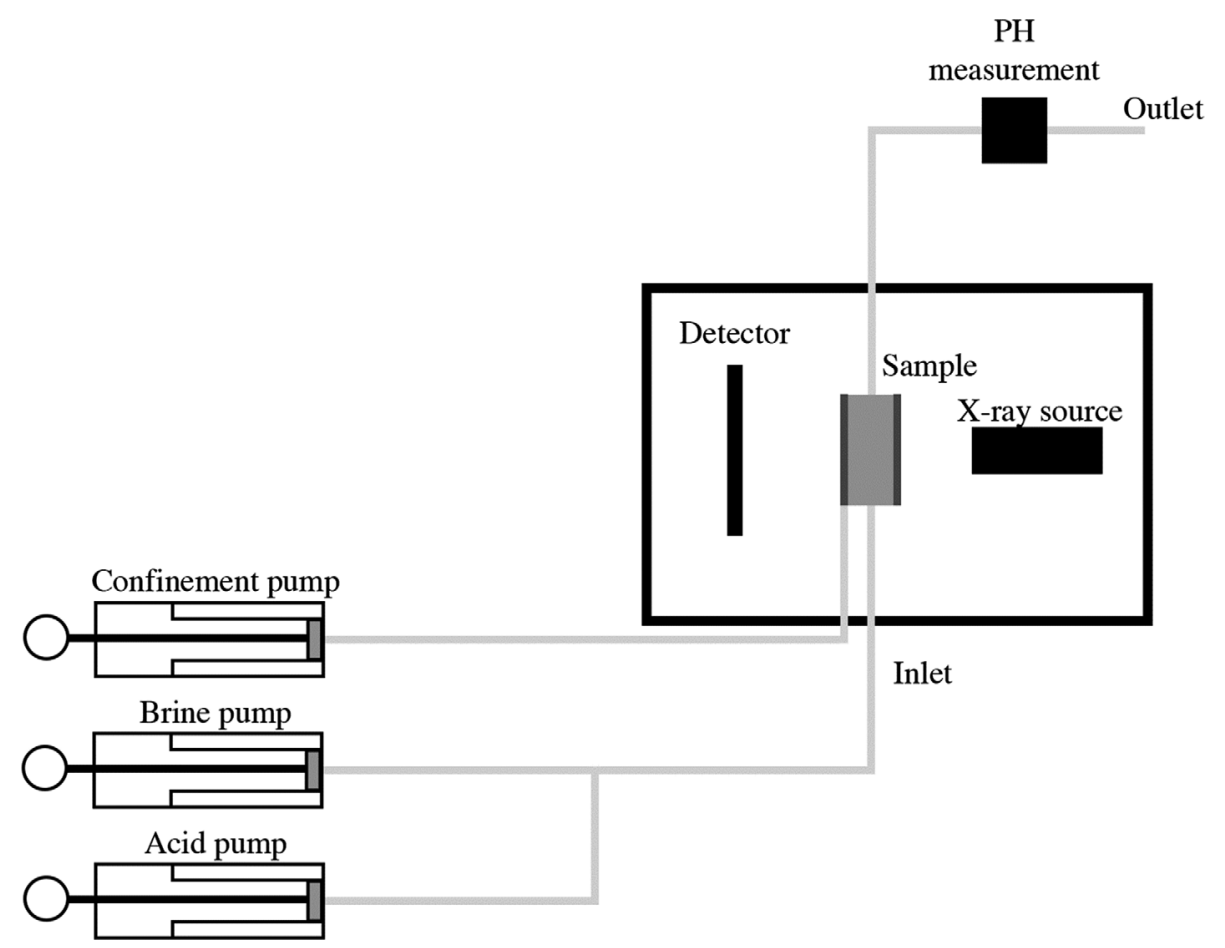

Fig. 4. Schematic overview of the scan and flow apparatus used for the calcite cement dissolution experiments.

A

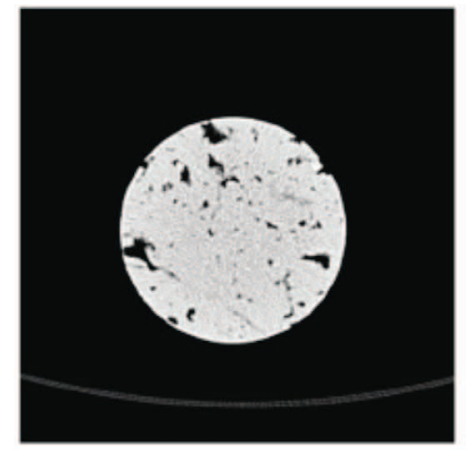

C

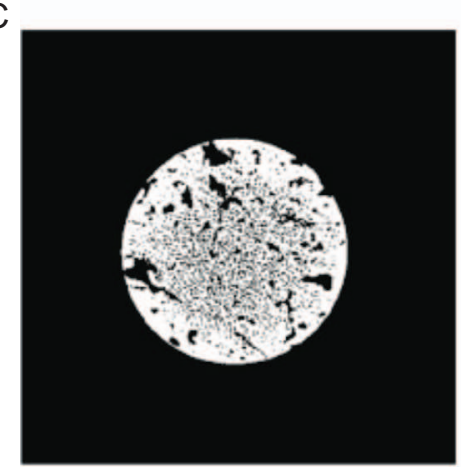

B

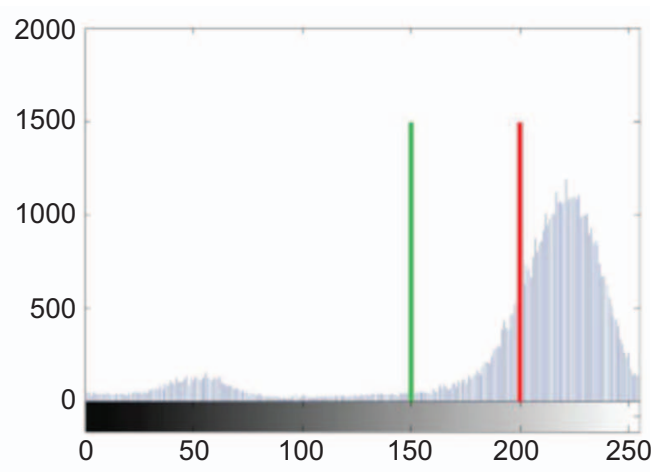

D

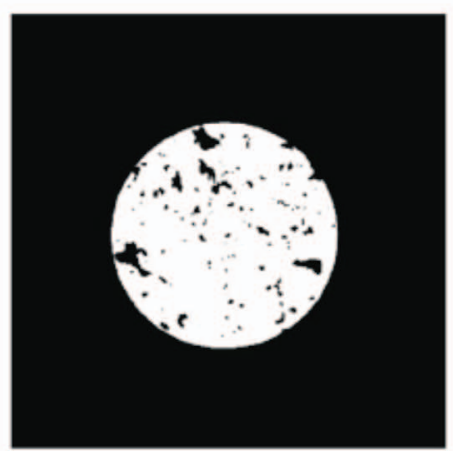

Fig. 5. Dual thresholding image analysis: A) original slice, B) histogram of the attenuation coefficients; the strong threshold is indicated in red; the weak threshold is indicated in green; C) matrix selection using only the strong threshold; D) resulting slice using both the strong and weak thresholds. 
A
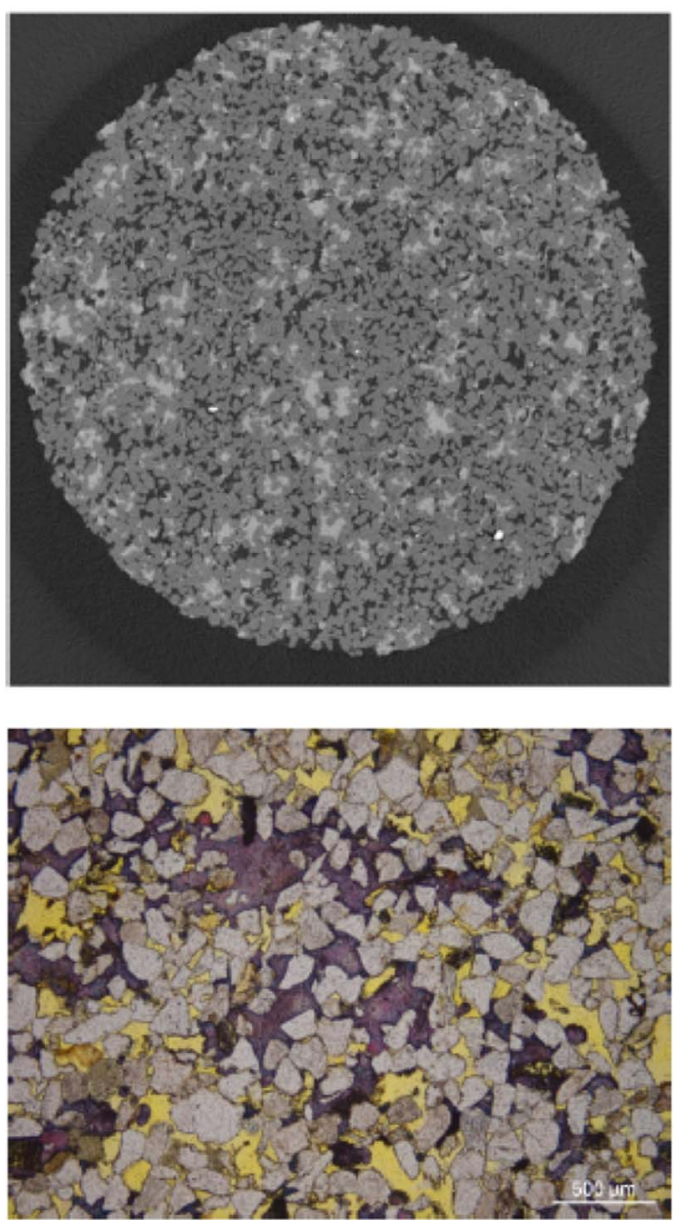

\section{B Heavy cemented}
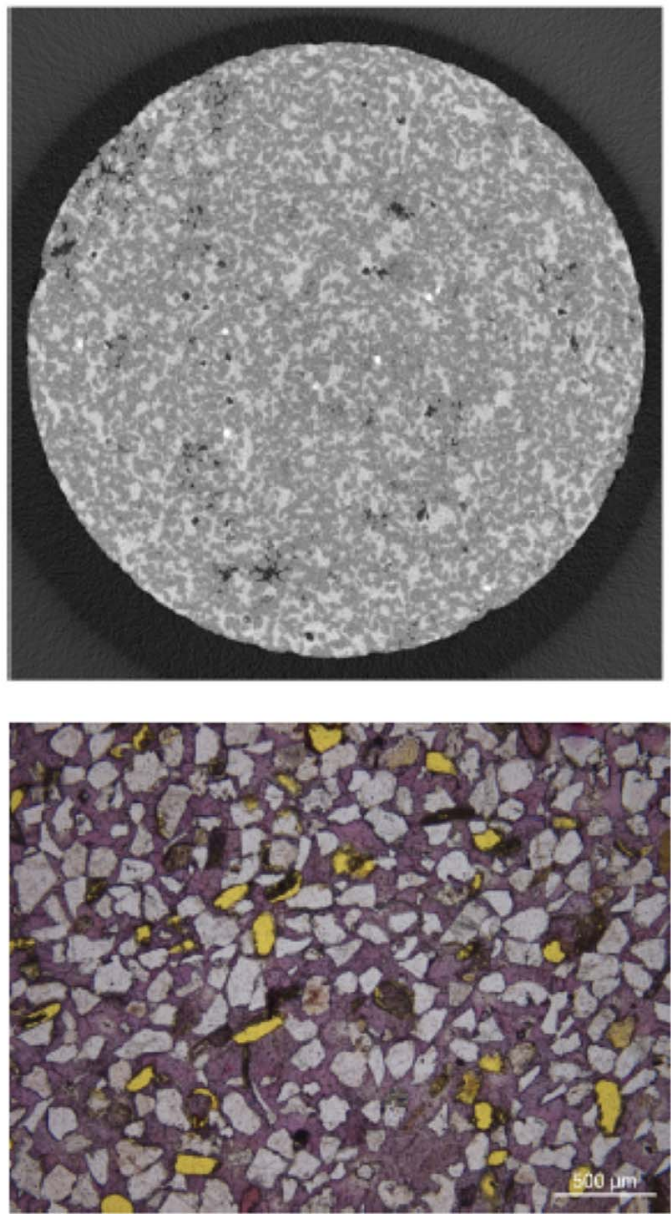

Fig. 6. Representative photomicrographs of the sandstone samples: A) CT slice and stained thin section of poorly cemented sandstone; B) CT slice and stained thin section of well cemented sandstone. Yellow represents remaining porosity.

noise in the dataset and the selection of less insulated foreground voxels (Claes, 2015).

\section{Results}

Samples are subdivided in three groups based on their percentage of cement content (as mentioned above). The first group consists of samples taken in the poorly cemented sandstone beds. The second group comprises samples originating from the heavily cemented lenses. A third group corresponds to the intermediate (transitional) cemented samples in between the first two groups. The difference in cement content between these groups can be clearly visualised on $\mathrm{CT}$ images and stained-thin sections (Fig. 6). The CT slices yielded good contrasting patterns allowing rapid identification of quartz, calcite and pore space and hence encouraging further petrographic investigations with Scanning Electron Microscope (SEM) and hot CL techniques (yet, not in the scope of this contribution). The photomicrographs presented in Figure 6 show the relative abundance of the calcite cement in the investigated sandstone samples. The purple colour of the stained calcite cement reflects its ferroan nature (Fe-rich calcite), which has been interpreted to be mostly of meteoric origin (Van Den Bril and Swennen, 2008).

Figure 7 represents plots of the porosity and cement percentages in function of the core height, based on the segmented CT slices for each group of samples. A third curve is featured to show the sum of the porosity and cement content, adding information about the original pore network before cementation occurred in the paragenesis. By comparing the two classes it becomes clear that the cemented samples have a tendency to be initially more porous compared to the less cemented ones. Figure $7 \mathrm{C}$ shows the sharp transition between the cemented and uncemented zones even on a $\mu \mathrm{m}$ scale, highlighting the heterogeneous characteristics of such calcite cementation on the investigated sandstone reservoir properties. 
A
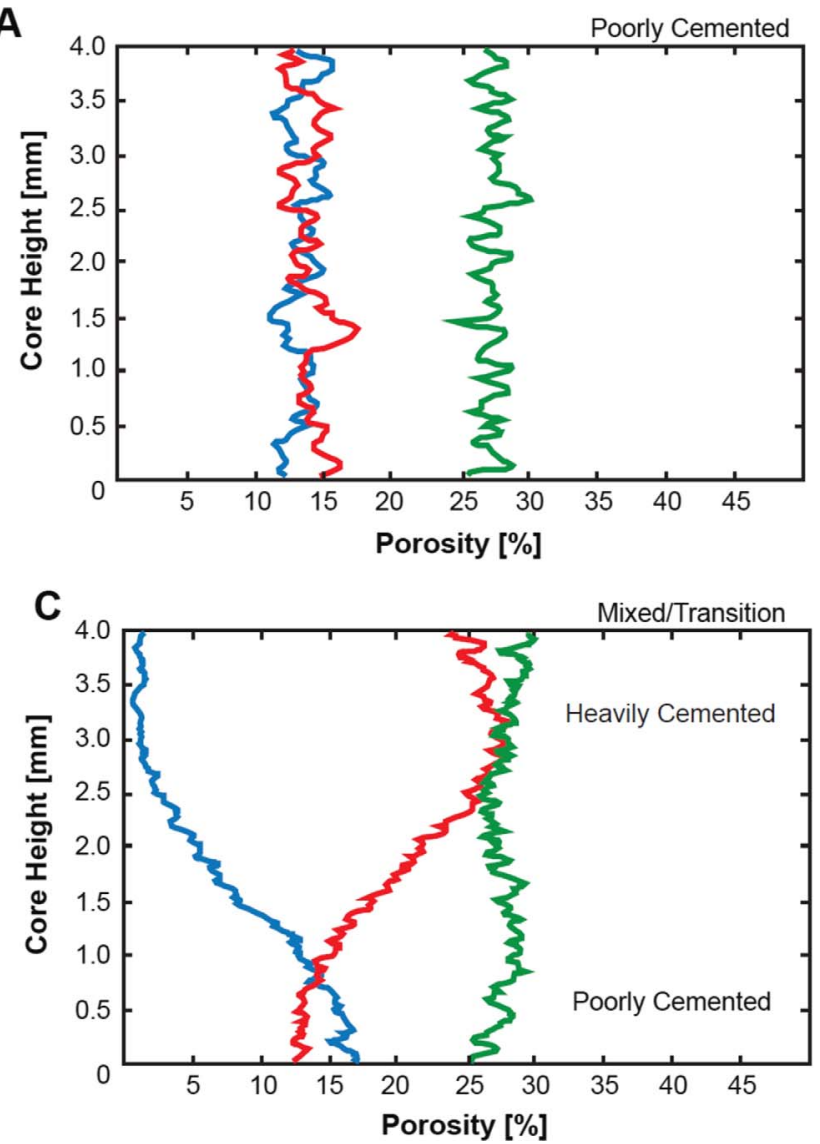

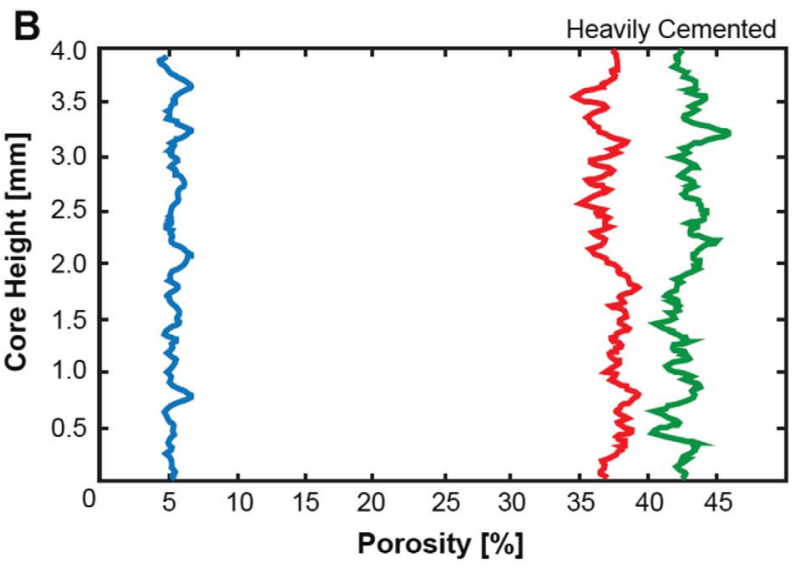

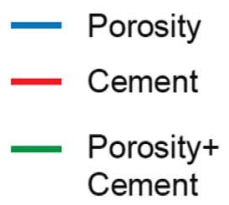

Fig. 7. Porosity(\%) and calcite cement(\%) curves along the height of the cored samples: A) poorly cemented sample; B) heavily cemented sample; C) transition sample.

\subsection{Representative Elementary Volume (REV)}

Heterogeneity in grain size, bio- and allochem compositions and pore distributions are general characteristics of reservoir rocks and relate to their geological history, i.e. sedimentary origin, burial history and diagenesis (Nader, 2016). These processes have an influence on the complexity of the pore network. However, when the scale of observation becomes large enough, the material can be viewed as a homogeneous continuum containing enough representative information of the heterogeneity. In order to address this problem, the concept of the REV (i.e. the smallest value that can be taken as a representation for the entire sample area/volume that does not respond to small changes in volume or location) was first introduced by Bear (1972).

The spatial distribution of lithological components needs to be represented in a numerical model in order to estimate reservoir properties, such as porosity. However computational limitations still restrict the number of heterogeneities taken into account in these models, especially on a sub-meter scale. Hence, by the use of the effective medium approximation, a hypothetical homogeneous property replaces a heterogeneous parameter (e.g. Claes, 2015). This indicates the importance of a correct estimation of the REV. Previous studies revealed the difficulty of establishing a general rule concerning the REV (Claes et al., 2016 and references therein). In this study the focus lays on geological samples, more specifically on the porosity parameter. The datasets used in this study provide an excellent starting point to analyze the heterogeneity of the pore network.

We use the assumption that the conditions essential for geostatistical interpretations can be linked to the definition of the REV. Brown et al. (2000) elaborated the assumption suggested by Bear (1972) that a volume falls within the region of the REV if it can be treated as an ergodic stationary function $[Z(x, V i)]$ in the domain $\mathrm{R}$. This corresponds to a second order stationary function in $\mathrm{R}$, which can be expressed as:

$$
E\left[Z\left(x, V_{i}\right)\right]=\text { constant }
$$

and

$$
\operatorname{cov}\left[Z\left(x+h, V_{i}\right), Z\left(x, V_{i}\right)\right]=f(h) \rightarrow 0 \quad \text { as } \quad h \rightarrow 0
$$

The first part of the requirement states that the expected value of $Z$ remains constant, while the second part demands that the covariance between two points is only function of the separation distance $h$ in all points $x$. This last requirement can be relaxed to the requirement expressed below if the sample consists of a finite number of observations from a continuous process, which is the case 
A
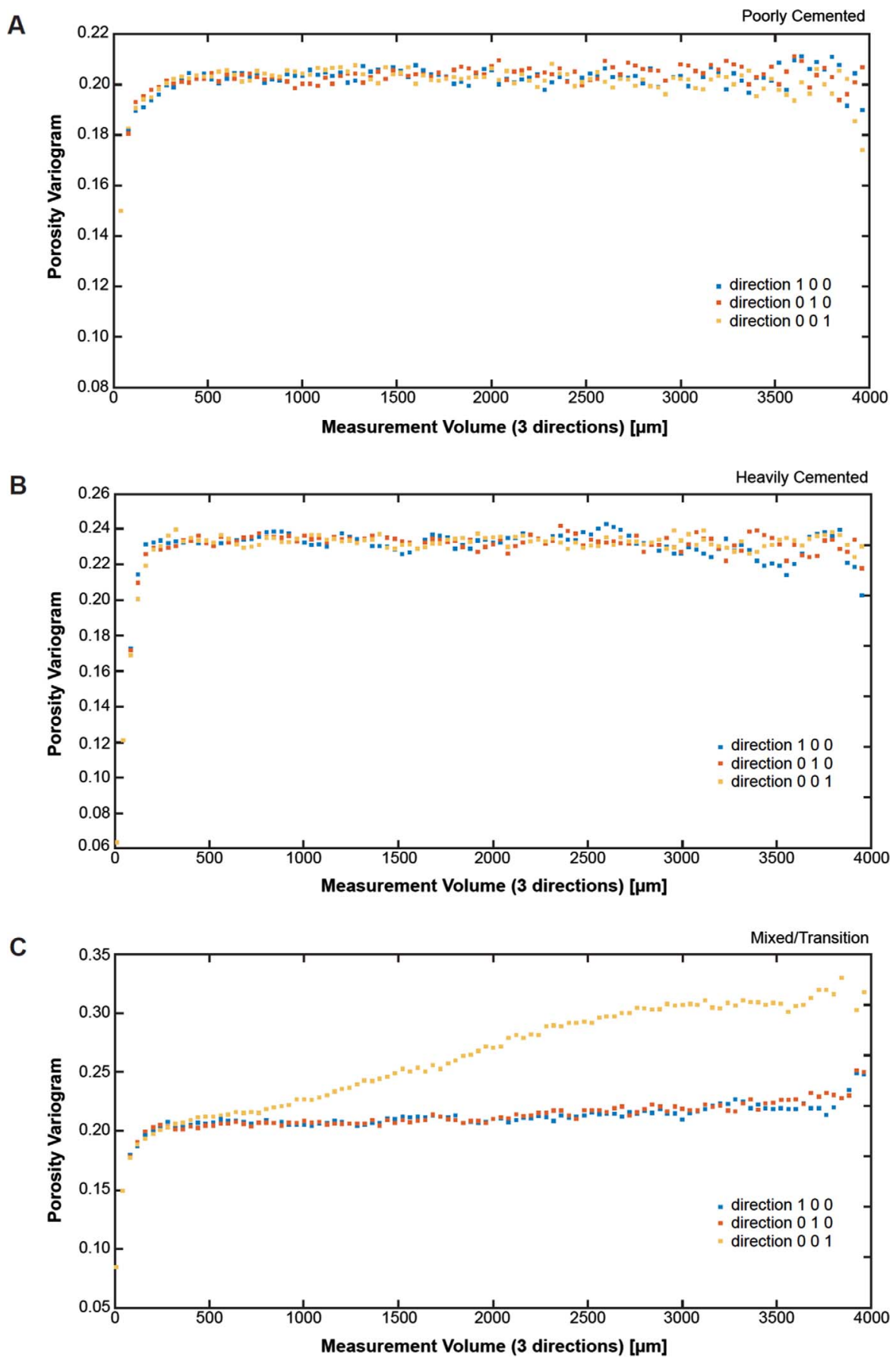

Fig. 8. Directional porosity variograms in the three principal directions: A) poorly cemented sample; B) heavily cemented sample, C) transition sample.

for the porosity parameter, according to Myers (1989) and Cressie (1992).

$$
\operatorname{Var}\left[Z\left(x, V_{i}\right)\right]=\text { constant }
$$

The semi-variogram is characterized by a sill and range (Fig. 8). The sill value represents the plateau value and is equal to the variance of the data. The range or length of correlation is the distance at which the sill value is reached. In this study, semi-variograms of the porosity parameter are calculated based on the segmented datasets (Fig. 8). Voxels representing the pore space have $a$-value of one, while the voxels of the rock phase are given $a$-value of zero. The obtained semi-variograms are achieved according to the spherical model, which has a linear behaviour near the origin and flattens out at larger distances (Isaaks and Srivastava, 1989).

The porosity REV for each sample group is shown in Figure 8 . The $3 \mathrm{D}$ volumes of the used plugs exceed the size 

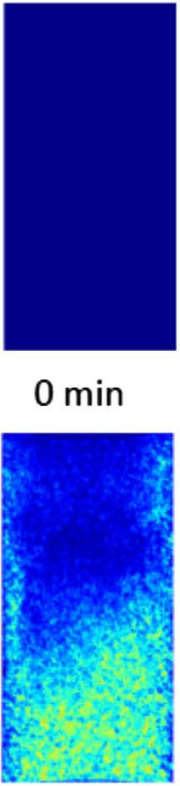

$150 \mathrm{~min}$

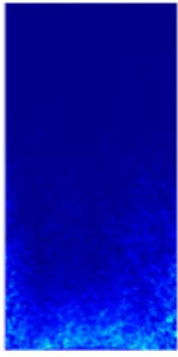

$30 \mathrm{~min}$

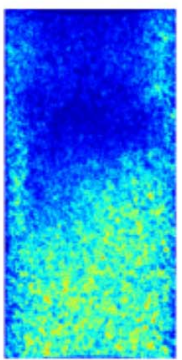

$180 \mathrm{~min}$
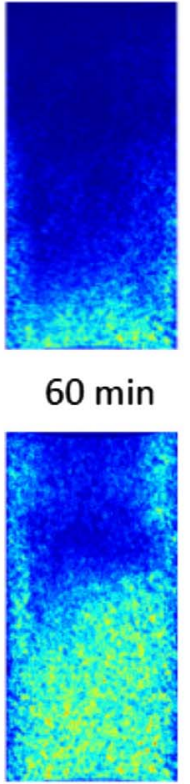

$210 \min$
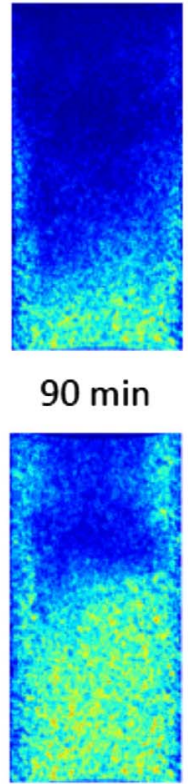

$240 \mathrm{~min}$
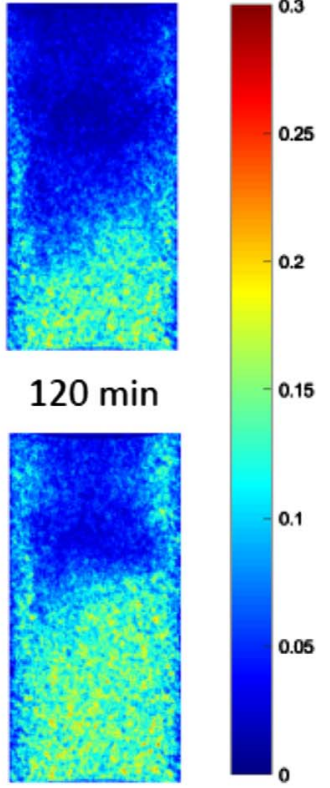

$270 \mathrm{~min}$

Fig. 9. Porosity increase caused by the dissolution of calcite cement during the experiment applied on the poorly cemented sandstone sample. The colour-scale bar represents the porosity increase (from 0 to $30 \%$ ).

of the REV for the poorly cemented and heavily cemented group. However, for the plugs containing the transition between poorly cemented and well cemented zones, the $\mathrm{REV}$ is larger than the used plug size. The heterogeneity of these samples becomes even clearer by calculating experimental semi-variograms in three orthogonal directions as illustrated in Figure 8. The REV of the poorly cemented and heavily cemented samples are in the same order of magnitude and vary between 300 and $500 \mu^{3}$.

\subsection{Dissolution experiments}

One representative sample of the poorly cemented sandstone and another sample of the heavy cemented group are used to perform the dissolution experiment - since both yielded acceptable REV. Changes of porosity and permeability in the studied plugs are attributed to the dissolution of the calcite cement. The dynamic processes are monitored by measuring the pressure difference between the inlet and outlet, the $\mathrm{PH}$ value of the fluid at the outlet as well as radiographs of the plug sample during the event. Because of the non-destructive measuring techniques used in the proposed experimental setup, both, the progression of the chemical reaction rate as well as the morphological changes in the porosity network can be quantified.

The principles of X-ray CT imaging can be used to calculate the porosity difference in consecutive radiographs based on the relationship expressed in Beer's law and that for composite materials, the contribution of each component can be calculated using a simple linear equation. The assumption is made that the only component changing the attenuation of the X-rays is the change in porosity. The accurate porosity measurements before and after the dissolution experiment are used to calibrate the linear relationship.

Figure 9 depicts the spatial change of the porosity value at different time intervals during the experiment. It is clear that a homogeneous propagation of the reaction front is obtained. The porosity increase starts at the bottom of the sample, where the acid is injected and spreads upwards. These results can be correlated with the total porosity, permeability and $\mathrm{PH}$ measurements during the experiment (Fig. 10). The overall porosity increases with time during the dissolution process. However, at the beginning of each experiment almost no porosity gain is observed. The porosity increase correlates with the main drop in the $\mathrm{PH}$ measurements. The governing principles behind this observation will be discussed below.

The results of the $\mathrm{PH}$ measurement/monitoring can also be used to determine the completion of the experiment on the poorly calcite cemented sandstone. Once the exiting fluid has the same $\mathrm{PH}$ value as the entering fluid, it becomes plausible to conclude that almost no reaction takes place anymore (i.e. the calcite cement was completely dissolved). Moreover, comparison of the central slice of the scan before and after experiment proves that almost all the cement has been indeed dissolved at the examined height (e.g. Fig. 11). The calculated increase in permeability is quite gradual, and corresponds well to the independent measured permeability at the end of the experiment. The final porosity value reached for the sample also corresponds to the initial segmentation of the CT results. At the end of the experiment, the remaining calcite cement was $1.5 \%(9 \%$ initially) and the measured porosity and permeability were $30.7 \%$ and $1300 \mathrm{mD}$, respectively $(21.5 \%$ and $374 \mathrm{mD}$, initially). 
A

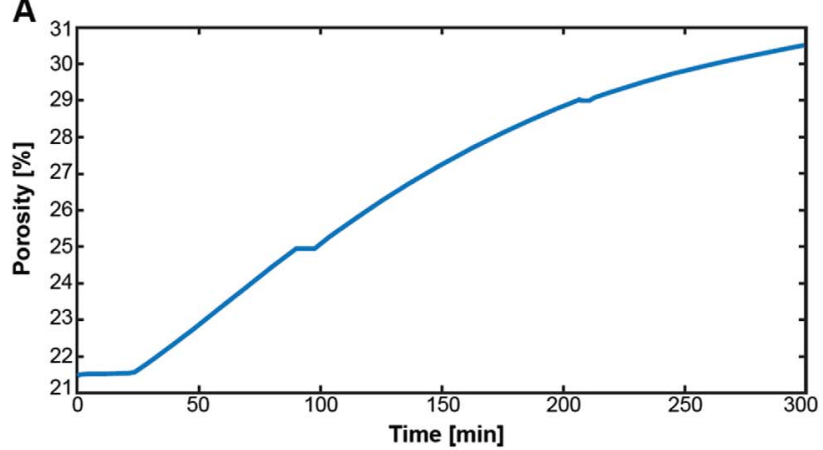

C

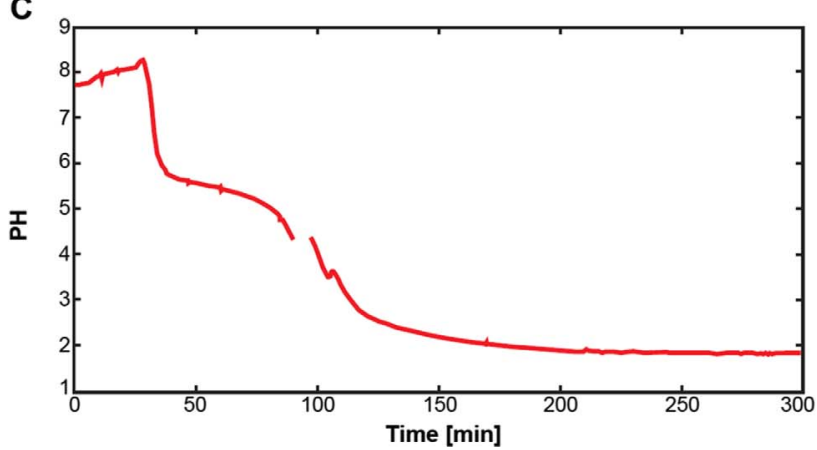

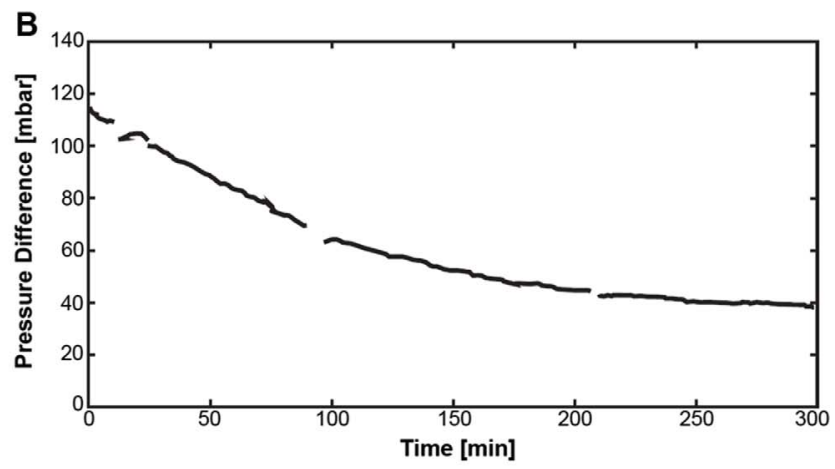

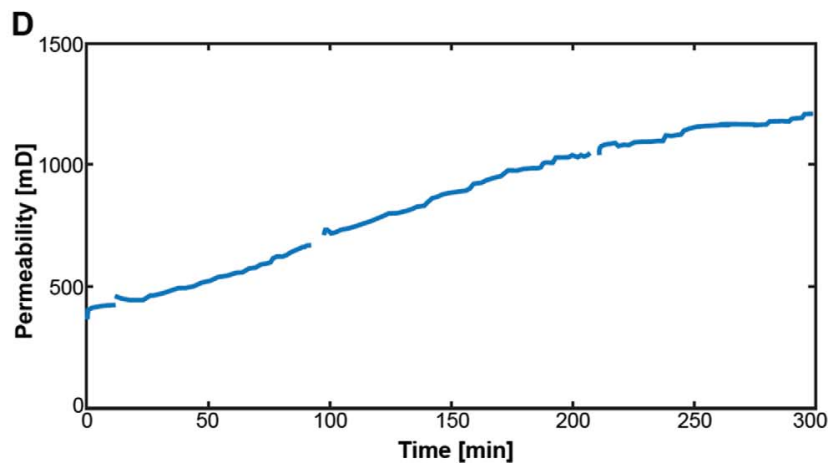

Fig. 10. Results of the cement dissolution experiment of the poorly cemented sample: A) porosity increase; B) pressure difference; C) $\mathrm{PH}$ value of the effluent flow; D) permeability increase.

A

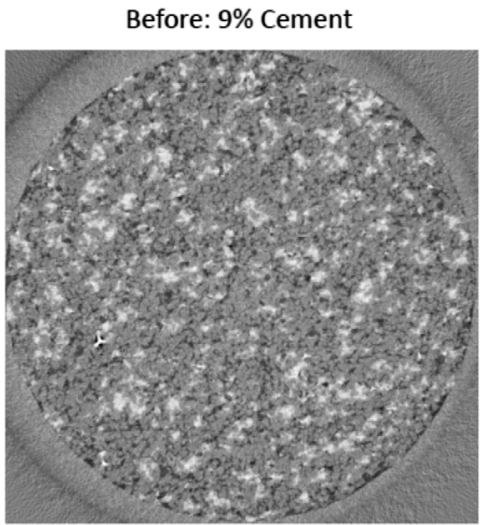

Poro: $21.45 \%$

Perm: 374 mD
B

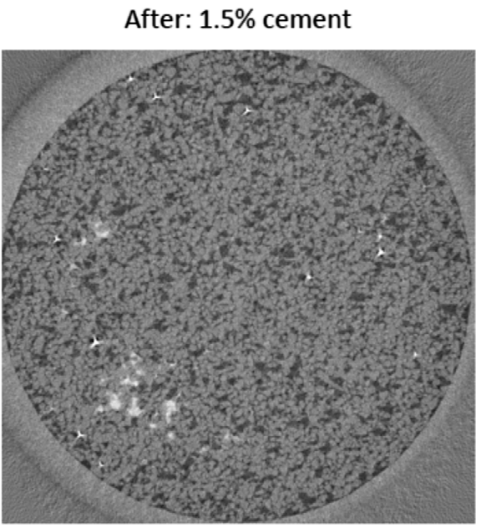

Poro: $30.72 \%$

Perm: 1300 mD

Fig. 11. CT slice and characteristic cement\%, porosity $\%$ and permeability values of the poorly cemented sample: A) before dissolution; B) after dissolution.

When the same workflow is applied on the heavily cemented sample, the outcome is clearly different as illustrated in Figure 12. No homogeneous dissolution occurs and a preferential pathway is formed instead. This is also reflected in a relatively smaller porosity increase compared to the less cemented sample. Moreover the duration of the experiment is much longer, indicating a much longer breakthrough timing although the same concentration of acid is used. This can be explained by the fact that the reaction needed to create a pathway and the acid became less powerful after it reacted with the cement. This is also reflected in the very high pressure difference in the sample compared to the previous experiment (Fig. 13).

These observations are also well illustrated by the resulting $\mathrm{PH}$ curve (Fig. 13C). When analysing the $\mathrm{PH}$ curve the same shape of the curve is observed as in the previous experiment. This is somewhat surprising because a lot of cement, which can react with the acid, is still present in the sample. After approximately $350 \mathrm{~min}$ - since the start of the experiment - the $\mathrm{PH}$ at the top of the sample becomes the same as at the bottom of the sample, suggesting very little reaction taking place in the sample at this stage. 

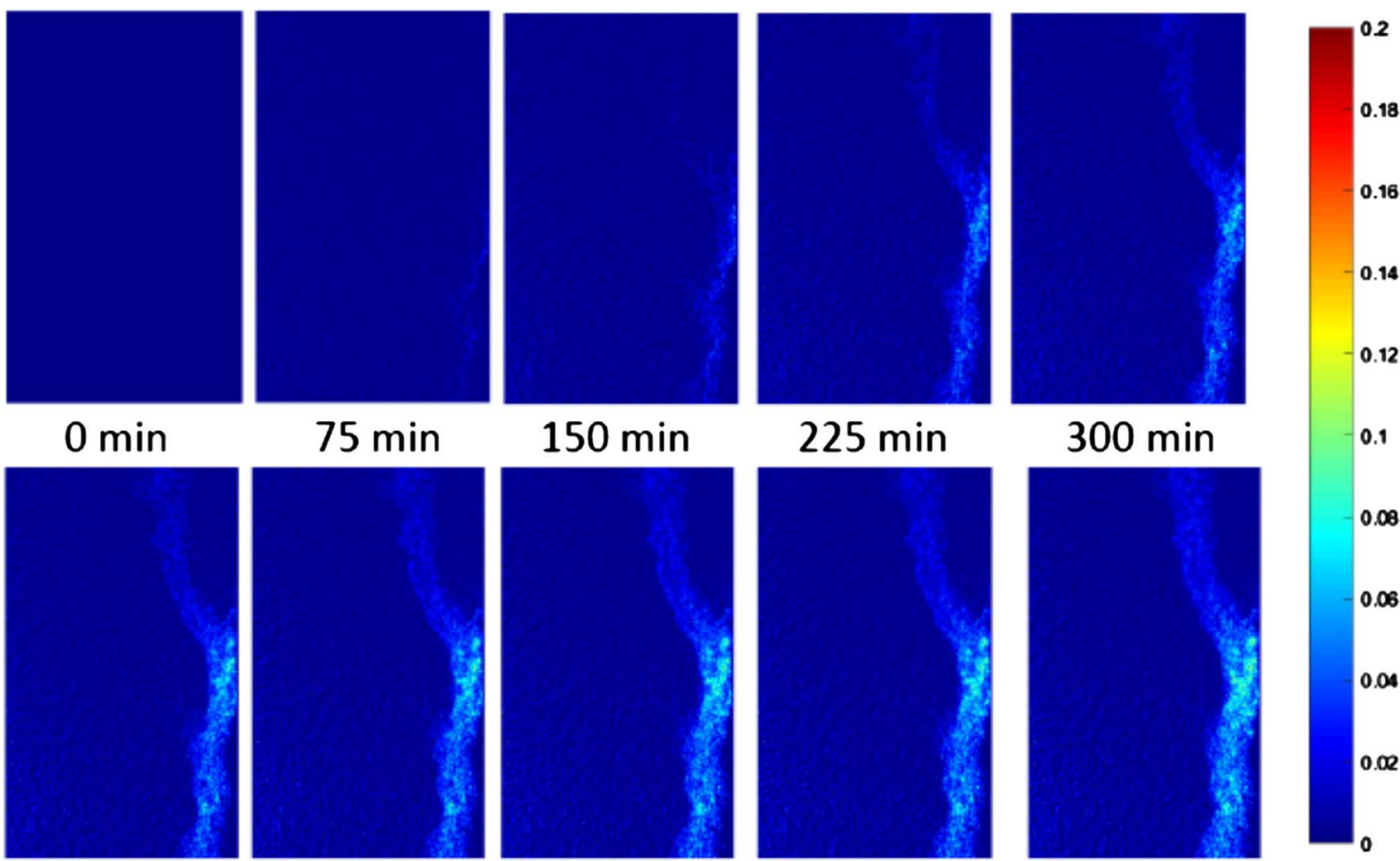

$675 \mathrm{~min}$

Fig. 12. Porosity increase caused by the dissolution of calcite cement during the experiment applied on the well cemented sandstone sample. The colour-scale bar represents the porosity increase (from 0 to $20 \%$ ).
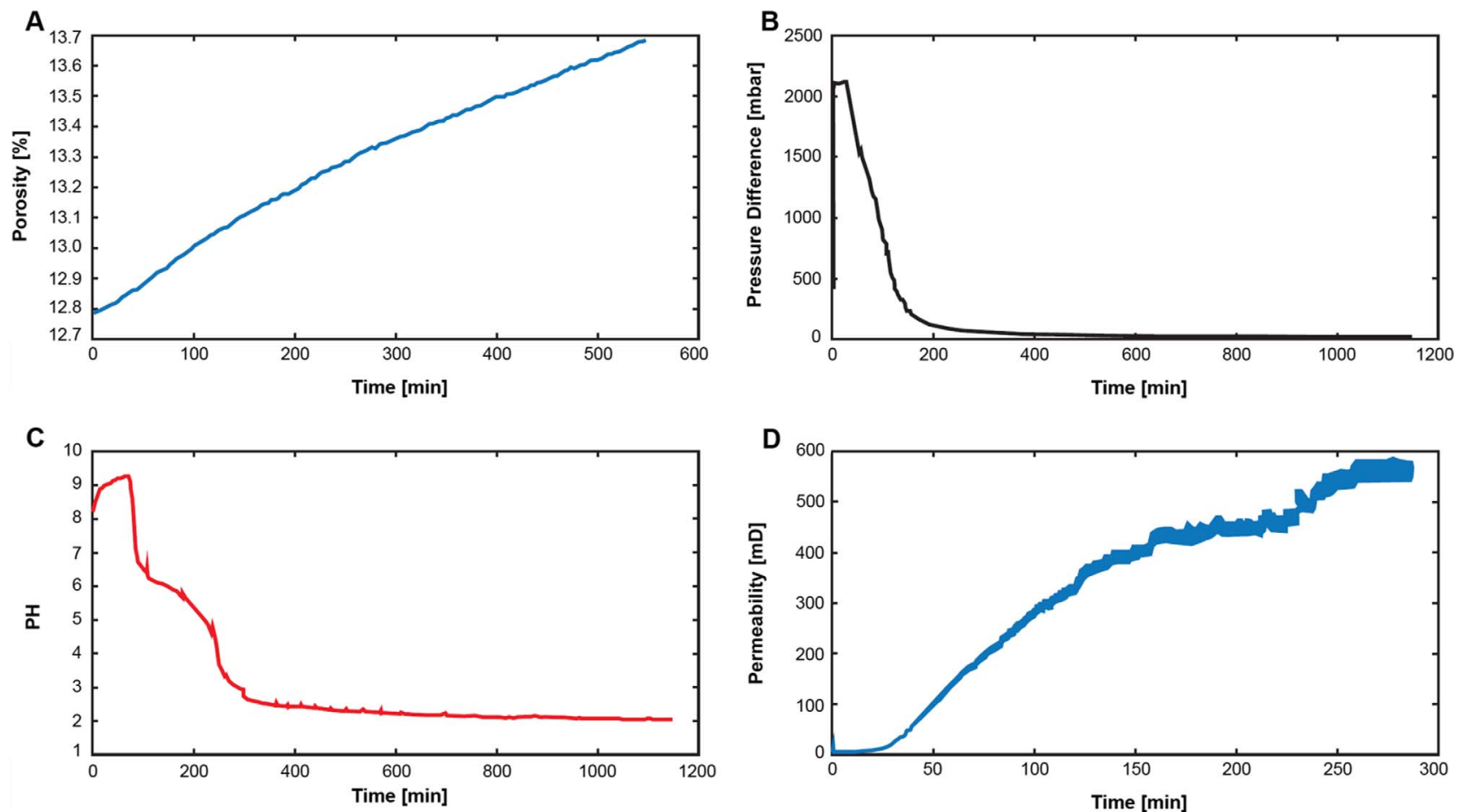

Fig. 13. Results of the cement dissolution experiment of the heavily cemented sample: A) porosity increase; B) pressure difference; C) $\mathrm{PH}$ value of the effluent flow; D) permeability increase. 
A

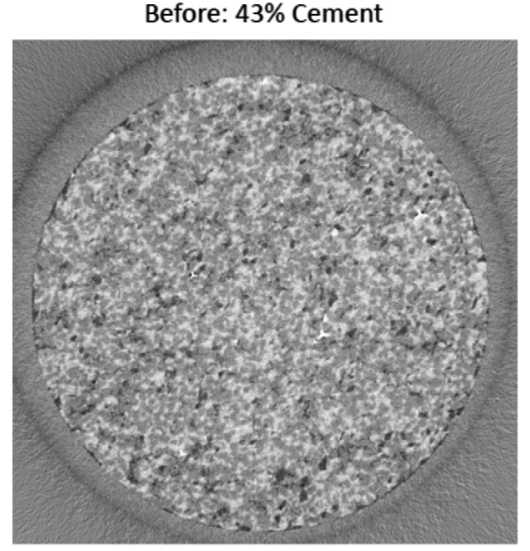

Poro: $12.55 \%$

Perm: $5.63 \mathrm{mD}$
B

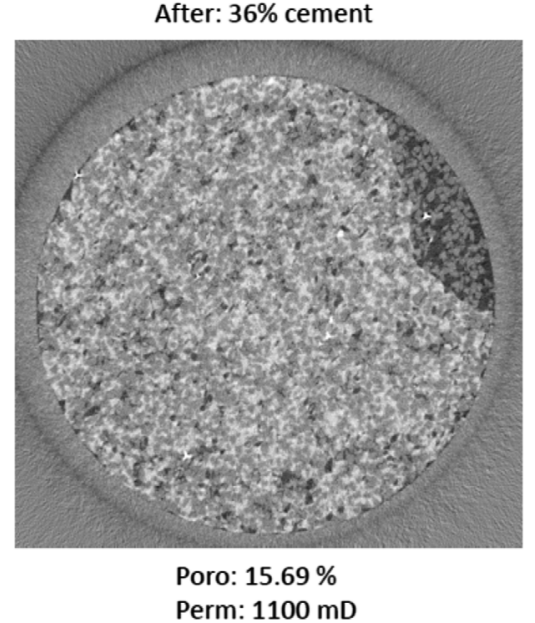

Fig. 14. CT slice and characteristic cement\%, porosity\% and permeability values of the heavily cemented sample: A) before dissolution; B) after dissolution.

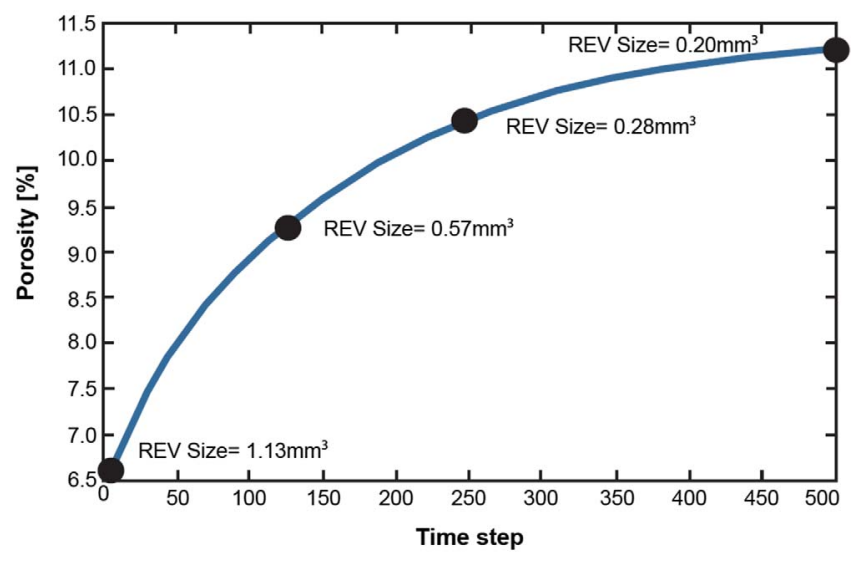

Fig. 15. REV sizes corresponding to simulated porosity increase due to homogeneous dissolution.

At the end of the experiment, the remaining calcite cement was as high as 36\% (43\% initially; Fig. 14). Measured porosity was about $16 \%$, compared to the initial $\sim 13 \%$, showing slight changes in the above all bulk of sample porosity. Yet, the permeability increased from $5.63 \mathrm{mD}$ to $1100 \mathrm{mD}$, illustrating indeed the worm-hole channel observed in Figure 12.

\section{Discussion}

\subsection{Implications on REV}

In order to test the influence of the dissolution experiment on the size of the REV, numerical simulations were performed. In a segmented CT volume of $500 \times 500 \times 500$ voxels the amount of cement was decreased layer by layer. Figure 15 depicts the observed porosity increase together with the corresponding REV size decrease. This decrease of REV size can be explained by the complexity reduction of the sample.
By reducing the cement (which results in increasing porosity) the number of components of the sample decreases. Therefore, the complexity and heterogeneity of the sample are reduced allowing lower REVs.

The results of this study confirm the existence of different REV sizes at different scales corresponding to different geological length scales (Norris et al., 1991; Nordahl and Ringrose, 2008). This is an important observation because it has major implications for reservoir modelling. Figure 16A shows a conceptual sketch of how the measured porosity can vary in function of the measured sample size. In this model it is important to notice that the porosity parameter will always level out at a higher porosity value than the previous REV value. This concept is added to the model because the porosity parameter can be viewed as a cumulative parameter. For example, the first attained REV can be controlled by intra-particle or intercrystaline porosity. If the sample becomes larger the addition of inter-particle or moldic porosity can result in a new REV for a higher porosity value. Finally, on a third level, the addition of vuggy porosity can introduce a new, larger REV. However, if the scales of heterogeneities are overlapping, it becomes almost impossible to suggest different sample sizes in order to completely characterize the examined parameter.

In this contribution, relatively simple cases of sandstone reservoirs with varying amounts of cement-reduced intergranular porosity (or inversely, cement-dissolution enhanced intergranular porosity) are investigated. The size of the REV is demonstrated to be influenced by the amount of cement present in the studied sample. Figure 16B depicts a schematic representation of the relationship between the cement content, REV size and geological time frame. For the sandstones that were uncemented as well as those that were well cemented, the REV sizes are relatively small and therefore, the average values can be safely upscaled. Yet, this is not applicable for partially cemented (transition) sandstones, whereby the measured REV (check also Fig. 8C) is larger than the investigated sample volume. For these heterogeneous samples, REV cannot be estimated 
A

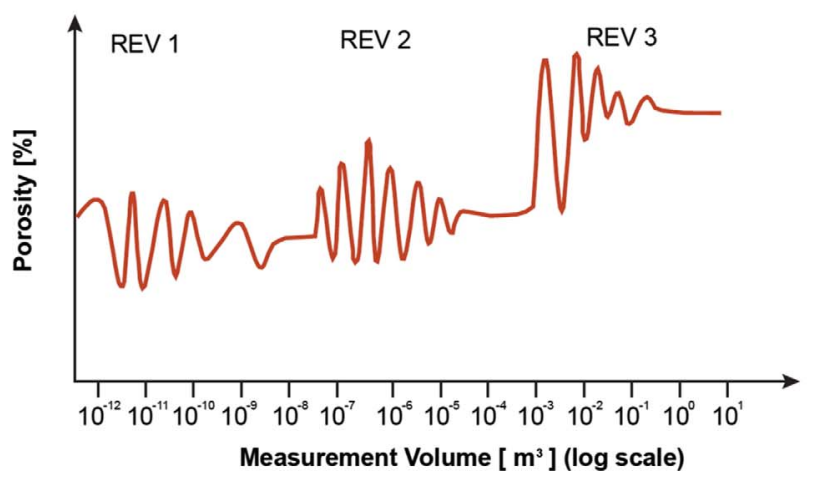

B

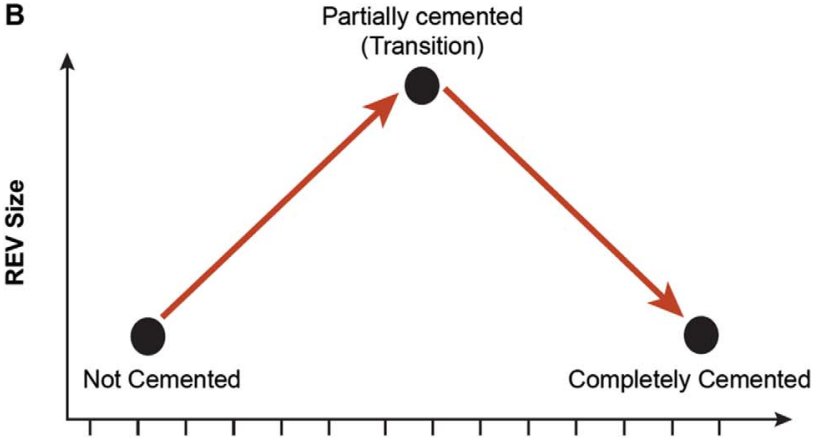

Diagenesis / Time (\% Cement / min)

Fig. 16. A conceptual sketch of different REVs reached at different sample volumes (A), and the effect of diagenetic components (cement\%) on the REV size (B).

A

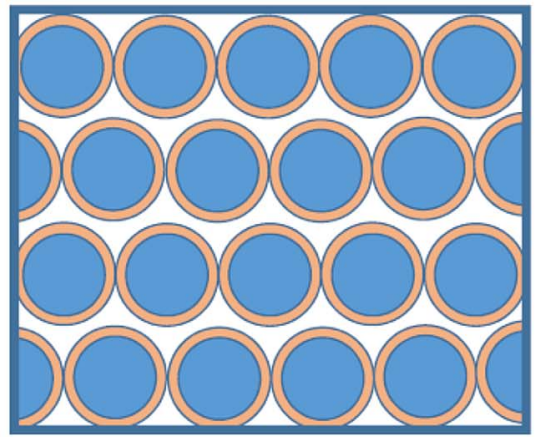

B

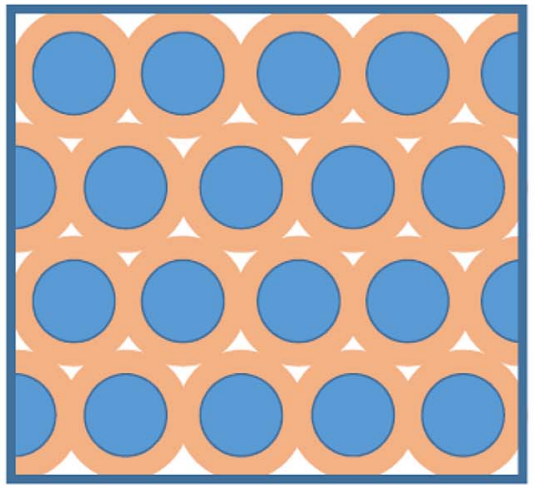

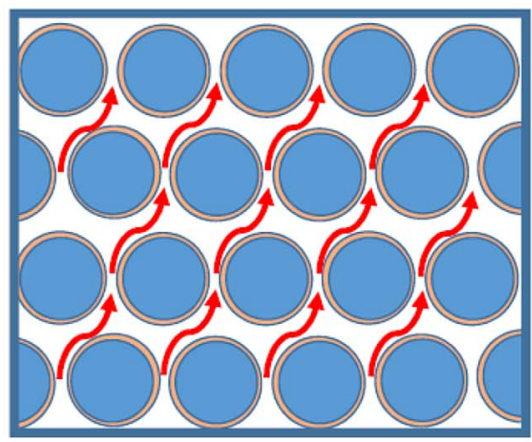

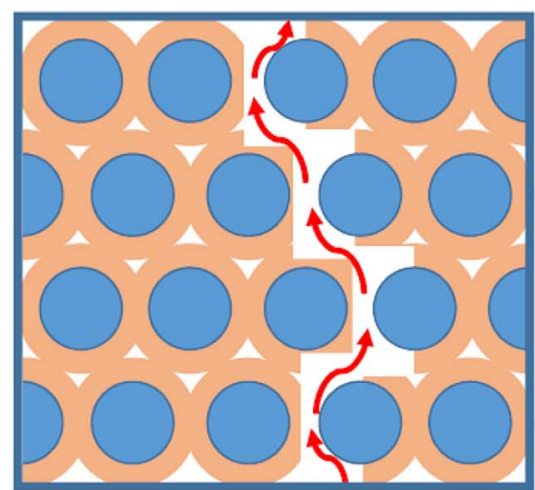

Fig. 17. Conceptual illustrations of different dissolution regimes in the poorly cemented and heavily cemented sandstones samples (A and B, respectively). White represents porosity, orange represents cement and blue are grains.

and other means for upscaling are needed. Such observations have also important consequences for reservoir modelling. In addition, a detailed knowledge of the paragenetic sequence is of uttermost importance to estimate the size of the REV.

\subsection{Interpretation of the worm-hole formation}

Figure 17 depicts schematic illustrations of the uniform dissolution and channel (worm-hole) formation concepts. The flow velocity distribution over the entire sample appears to be narrower for the uniform case (Fig. 17A). Hence we can assume that the flow speeds are also uniform and relatively high. The reaction products moved away efficiently from the surface of the sand grains in the created advective flow. Hence, fresh reactive fluid is continually exposed to the entire rock surface. This results in the uniform observed reaction front (cf. Fig. 9). Over $1 / 3$ of the experiment duration the effluent $\mathrm{PH}$ remains high as the $\mathrm{Ca}^{2+}$ ions are represented for the entire pore fluid in the sample.

During the experiment, when the channelling prevailed, the velocity distribution spanned many orders of 
magnitude and was not be homogeneously distributed in the sample (Fig. 17B). Approximately $80 \%$ of the sample volume did not contribute to the experiment. Hence the majority of the injected fluid passed through the created channel without further reacting with the cement present in the sample elsewhere (cf. Fig. 12). This problem could potentially be solved by subjecting the sample to different velocity fields. These observations correspond to the ones made by Menke et al. (2017). Although this approach was not achieved in this present contribution, it could consist in a rewarding research project in perspective.

\subsection{Relationships with diagenesis processes}

Different early diagenetic processes can be grouped in either advection controlled or diffusion controlled processes (Berner, 1980). Walderhaug and Bjørkum (1998) suggested that the nucleation site distribution can be used to infer whether a cementation process was dominantly controlled by advection or diffusion. In the early diagenetically cemented lenses of the Luxembourg sands, the carbonate fragments which acted as nucleation sites for the first cement generation were located close to each other (Van Den Bril and Swennen, 2008), which implies that cementation likely was controlled by advection. The most probable source and process to initiate such early cementation relate to saturated seawater flow through the sediment, which was also suggested by Molenaar (1998). The fact that seawater was saturated with respect to calcite is evidenced by the sometimes high amounts of fibrous ooids in the sediment (Van Den Bril and Swennen, 2008). Possible mechanisms for pore water flow relate to storm and wave activity or currents created by tidal activity (tidal-pumping). The latter mechanism mainly affects the upper $2-4 \mathrm{~m}$ of the sedimentary succession in coastal areas on the continental shelf.

To initiate cementation, in general, several conditions have to be fulfilled, namely: (i) existence of a calcite (over)saturated fluid, in this case seawater; (ii) a source of $\mathrm{Ca}^{2+}$ and $\mathrm{HCO}^{3-}$, in this case unstable detrital carbonate; and (iii) presence of carbonate nucleation sites. The latter two conditions address the influence of detrital composition on the diagenetic evolution. Two additional parameters which control the geometry of cemented zones are: (i) permeability variations creating localized fluid flow; (ii) residence time within the influence of fluid flow. Point counting indicates that these cements are volumetrically more abundant in coarse grained sections than in the very fine-grained cemented layers. Cementation started in the centers of the cross-stratified lenses where high amounts of carbonate fragments occurred. The latter most likely were also most porous zones as shown by the porosity measurements. Variation in flow velocity during deposition of the sands resulted in subtle grain size variations as well as sorting and small compositional variations (Hartkamp et al., 1993). This variability can even be seen on the scale of a thin section. The early calcite cement was followed by a later, coarser calcite cement (ferroan phase), which is believed to be of meteoric origin (Van Den Bril and
Swennen, 2008). The latter cement appears to be more abundant and impacts principally the porosity of the host sandstone reservoirs.

The presented calcite dissolution experiments of poorlyand well-cemented sandstones, illustrated the inverse process of cementation. A process that can affect these sandstones or equivalent (analog) reservoirs before charging with hydrocarbon and/or after underground storage of fluids (e.g. $\mathrm{CO}_{2}$, gas). Understanding the formation and behavior of reservoirs in quantitative and representative ways is vital for both hydrocarbon exploration and production as well as subsurface storage and management. This contribution presents a case study and a workflow that can be applied in order to better quantify the effect of diagenetic processes such as dissolution (and inversely, cementation) on reservoir properties, questioning also the representativity of the analyses and the impact on proposed REV for an upscaling strategy. The way forward is to apply such workflow for reservoir-scale modelling and use the quantified diagenetic phases (e.g. cement\%, dissolution/porosity\%) within the concept of REV to homogenize and upscale meaningfully the flow properties. Another challenging perspective would associate several diagenetic pore-space altering processes within a paragenetic trend and quantify their combined effects on the reservoir properties.

\section{Conclusion}

The overall goal of this research is to come up with a more accurate description and characterization of the pore structure of geological samples. Computer Tomography (CT) coupled with a flow apparatus and numerical solutions proved to be a successful approach for achieving this objective. Because of its inherent 3D data acquisition, the complete internal structure of the scanned sample can be investigated at different resolutions. The visualization of the connectivity of the pore network and the detection of additional phases result in a detailed characterization of the geological samples. The generation of 3D datasets also permits a more quantitative description and calculation of different important parameters such as porosity, permeability and cement content.

This new approach can quantify the influence of diagenetic phases (e.g. dissolution, cement plugging) on flow properties (porosity and permeability) of reservoir rocks. Representative types of Early Jurassic, Luxembourg Sandstone Formation samples with different diagenetic characteristics (amount of calcite cement) were used. The results show a distinctive dissolution pattern for different cementation grades. In addition, the evolution of the diagenetic process (i.e. dissolution) and its impact on the flow properties with time (during the experiment) were adequately quantified. Such results may also provide further constraints for geochemical reactive transport modelling, and pave the way for meaningful upscaling of flow properties through the concept of diagenetic REV for reservoir modelling. The same approach can be applied on other type of cements and host-rocks, and could be improved by integrating other petrophysical analyses (e.g. petroacoustic, NMR). 
Acknowledgments. The authors want to thank Prof R. Swennen and Dr K. Van Den Bril for providing the samples used in this study. Their support is kindly acknowledged. The two reviewers and the OGST Editorial office are also thanked for their constructive comments and suggestions which helped in improving the content and form of this work.

\section{References}

Algive L., Bekri S., Nader F.H., Lerat O., Vizika O. (2012) Impact of diagenetic alterations on petrophysical and multiphase flow properties of carbonate rocks using a reactive pore network modelling approach, Oil Gas Sci. Technol. - Rev. IFP Energies nouvelles 67, 1, 147-160.

Allen J.R.L. (1980) Sand waves - a model of origin and internal structure, Sediment. Geol. 26, 281-328.

Bear J. (1972) Dynamics of fluids in porous media, American Elsevier, New York.

Berner R.A. (1980) Early diagenesis: A theoretical approach.Princeton series in geochemistry. Princeton University Press, Princeton. 241 p.

Berners H.P. (1985) Der Einfluss der Siercker Schwelle auf die Faziesverteilungen meso-känozoischer Sedimente im NE des Pariser Beckens: Ein Sedimentationsmodell zum Luxemburger Sandstein (Lias), spezielle Aspekte zur structurellen Änderung der Beckenconfiguration und zum naturräumlichen Potential, PhD Dissertation, Rheinisch-Westfälischen Technische Hochschule, Aachen, 321 p.

Bintz J., Muller A. (1965) Sur la Représentation du "Grès de Luxembourg" sur la nouvelle carte géologique générale du Grand-Duché, Arch. Inst. G.-D. Luxembourg - Sect. Sc Nat. XXX1, 241-258.

Brown G.O., Hsieh H.T., Lucero D.A. (2000) Evaluation of laboratory dolomite core sample size using representative elementary volume concepts, Water Resour. Res. 36, 1199-1207.

Claes S. (2015) Pore classification and upscaling strategy in travertine reservoir rocks, PhD Thesis, KU Leuven, Leuven, Belgium, $119 \mathrm{p}$.

Claes S., Soete J., Cnudde V., Swennen R. (2016) A 3D classification for mathematical pore shape description in complex carbonate reservoir rocks, Math. Geosci., 48, 6, 619-639, https://doi.org/10.1007/s11004-016-9636-z

Cressie N. (1992) Statistics for spatial data, Terra Nova 4, 613-617.

De Boever E., Varloteaux C., Nader F.H., Foubert A., Bekri S., Youssef S., Rosenberg E. (2012) Quantification and prediction of the 3D pore network evolution in carbonate reservoir rocks, Oil Gas Sci. Technol. - Rev. IFP Energies nouvelles 67, 1, 161-178.

Deschamps R., Kohler E., Gasparrini M., Durand O., Euzen T., Nader F.H. (2012) Impact of mineralogy and diagenesis on reservoir quality of the Lower Cretaceous Upper Mannville Formation (Alberta, Canada), Oil Gas Sci. Technol. - Rev. IFP Energies nouvelles 67, 1, 31-58.

Dickson J.A.D. (1966) Carbonate identification and genesis as revealed by staining, J. Sediment. Petrol. 36, 2, 491-505.

Gibbons K., Hellem T., Kjemperud A., Nio S.D., Vebbenstad K. (1993) Sequence architecture, facies development and carbonate-cemented horizons in the Troll Field reservoir, offshore Norway, in: Ashton M. (ed.), Advances in reservoir geology,
Geological Society, London, Special publications, 69, pp. $1-31$.

Hartkamp C.A., Arribas J., Tortosa A. (1993) Grain-size, composition, porosity and permeability contrasts within cross-bedded sandstones in tertiary fluvial deposits, central Spain, Sedimentology 40, 787-799.

Isaaks E.H., Srivastava R.M. (1989) An introduction to applied geostatistics, Oxford University Press, New York, 561 p.

Menke H.P., Bijeljic B., Blunt M.J. (2017) Dynamic reservoircondition microtomography of reactive transport in complex carbonates: Effect of initial pore structure and initial brine pH, Geochim. Cosmochim. Acta 204, 267-285.

Mertens G., Spies E.-D., Teyssen T. (1983) The Luxemburg Sandstone Formation (Lias), a tide-controlled deltaic deposit, Ann. Soc. Géo. Belgique 106, 103-109.

Molenaar N. (1998) Origin of low permeability calcite-cemented lenses in shallow marine sandstones and $\mathrm{CaCO} 3$ cementation mechanisms: an example from the Lower Jurassic Luxemburg Sandstone, Luxemburg, in: Morad S. (ed.) Carbonate cementation in sandstones, Special publication of the International Association of Sedimentologists, 26, Blackwell Science, Cambridge, pp. 193-211.

Monteyne R. (1960) Les stratifications obliques dans l' Hettangien et le Sinémurien belges, Bull. Soc. Belge Géol. 69, 42-47.

Mostaghimi P., Blunt M.J., Bijeljic B. (2013) Computations of absolute permeability on micro-CT images, Math. Geosci. 45, 103-125.

Myers D.E. (1989) To be or not to be... stationary? That is the question, Math. Geol. 21, 347-362.

Nader F.H. (2016) Multi-scale quantitative diagenesis and impacts on heterogeneity of carbonate reservoir rocks, Springer, ISBN: 978-3-319-46444-2, 146 p.

Nordahl K., Ringrose P.S. (2008) Identifying the representative elementary volume for permeability in heterolithic deposits using numerical rock models, Math. Geosci. 40, $753-771$.

Norris R., Lewis J., Heriot-Watt U. (1991) The geological modeling of effective permeability in complex heterolithic facies, Paper SPE 22692 presented at the 66th Annual SPE Technical Conference and Exhibition, 6-9 October, Dallas, TX, USA.

Prosser D.J., Daws J.A., Fallick A.E., Williams B.P.J. (1993) Geochemistry and diagenesis of stratabound calcite cement layers within the Rannoch Formation of the Brent Group, Murchison Field, North Viking Graben (northern North Sea), Sediment. Geol. 87, 139-164.

Remeysen K., Swennen R. (2008) Application of microfocus computed tomography in carbonate reservoir characterization: possibilities and limitations, Mar. Pet. Geol. 25, 486-499.

Roduit N. (2007) JMicroVision: un logiciel d'analyse d'images pétrographique polyvalent, $P h D$ Thesis, University of Geneva, Switzerland, Earth \& Environment, Vol. 65, ix +116 p. ISBN 2-940153-64-7.

Swanson B.F. (1981) A simple correlation between permeabilities and mercury capillary pressures, J. Pet. Technol. 33, 12, 2498-2504. SPE-8234-PA. https://doi.org/10.2118/8234-PA

Van Den Bril K., Swennen R. (2008) Sedimentological control on carbonate cementation in the Luxembourg Sandstone Formation, Geol. Belg. 12, 3-23.

Van der Land C., Wood R., Wu K., van Dijke M.I.J., Jiang Z., Corbett P.W.M., Couples G. (2013) Modelling the permeability evolution of carbonate rocks, Mar. Pet. Geol. 48, $1-7$. 
Walderhaug O., Bjørkum P.A. (1998) Calcite cement in shallow marine sandstones: growth mechanisms and geometry, in S. Morad (ed), Carbonate cementation in sandstones, Special publication of the International Association of Sedimentologists, Blackwell Science, Cambridge, 26, pp. 179-192.

Youssef S., Deschamps H., Dautriat J., Rosenberg E., Oughanem R., Maire E., Mokso R. (2013) 4D imaging of fluid flow dynamics in natural porous media by ultra-fast X-ray microtomography, Paper SCA 2013 presented at the International Symposium of Core Analysts, 16-19 September, Napa Valley, CA, USA.
Youssef S., Han M., Bauer D., Rosenberg E., Bekri S., Fleury M., Vizika O. (2008) High resolution $\mu$-CT combined to numerical models to assess electrical properties of bimodal carbonates, Paper SCA 2008 - Temp Paper \#A54 presented at the International Symposium of Core Analysts, 29-30 October, Abu Dhabi, UAE.

Youssef S., Rosenberg E., Gland N., Skalinski M., Vizika O. (2007) High resolution CT and pore-network models to assess petrophysical properties of homogeneous and heterogeneous carbonates, Paper SPE 111427 presented at the SPE/EAGE Reservoir Characterization and Simulation Conference, 28-31 October, Abu Dhabi, UAE. 